\title{
Immediate continuous passive motion after internal fixation of an ankle fracture
}

\author{
Pasquale Farsetti $\cdot$ Roberto Caterini · \\ Vito Potenza · Vincenzo De Luna · \\ Fernando De Maio · Ernesto Ippolito
}

Received: 8 December 2007 / Accepted: 16 February 2009/Published online: 13 March 2009

(C) Springer-Verlag 2009

\begin{abstract}
Background Surgical treatment is usually mandatory in displaced bimalleolar and trimalleolar fractures. Some authors have recommended early mobilization of the ankle joint after surgical treatment of these lesions. In this study, we evaluate the effect of immediate postoperative continuous passive motion in the management of displaced bimalleolar and trimalleolar fractures treated surgically.

Materials and methods Two series of 22 patients each, who had had a Weber type A, B or C ankle fracture treated surgically, were followed up at least 10 years after the injury. In the first series, immediately after surgery, a continuous passive motion machine was applied to the operated ankle for 3 weeks, whereas in the second series, after surgery a plaster splint or a plaster cast was applied for 3 weeks.

Results At follow-up, all patients were evaluated clinically and radiographically using the AOFAS Ankle Hindfoot Score System (Kitaoka, Foot Ankle 15:349-353, 1994). The average final score for the first series of patients was 95.7 points (range $87-100$ points, standard deviation 3.42 points). Of this series, at radiographic examination, in two patients we observed minor signs of osteoarthritis of the ankle joint. The average final score for the second series was 88 points (range 68-100 points, standard deviation 10.60 points). At radiographic examination, in six patients we observed minor signs of osteoarthritis of the ankle joint, whereas in another one the osteoarthritis was severe.
\end{abstract}

P. Farsetti · R. Caterini · V. Potenza · V. De Luna ( $₫)$. F. De Maio - E. Ippolito

Divisione di Ortopedia e Traumatologia, Policlinico di Tor

Vergata, Università degli Studi di Roma "Tor Vergata",

Viale Oxford no 81, 00133 Rome, Italy

e-mail: videluna@tin.it
Conclusions Continuous passive motion started immediately after surgery seems to be an effective method both for allowing complete and quick recovery of the range of motion of the ankle and for reducing the risk of early degenerative joint disease. Immediate passive ankle motion can be applied only after adequate reduction and stable internal fixation.

Keywords Ankle - Bimalleolar · CPM therapy · Fracture · Trimalleolar

\section{Introduction}

Bimalleolar and trimalleolar fractures are extremely common in skeletal traumatology. Surgical treatment of these lesions is usually recommended when they are displaced, in order to obtain an adequate reduction and a stable internal fixation, and to avoid early degenerative disease of the ankle [2-4]. Conservative treatment is considered only in undisplaced fractures. Some authors report good results with surgical treatment and early mobilization of the ankle joint [5-8]; however, to the best of our knowledge, no papers have been published on the application of continuous passive motion (CPM) to the ankle immediately after surgery. In this study, we compared two series of 22 patients each, with displaced bimalleolar and trimalleolar fractures treated by open reduction and internal fixation and followed up at least 10 years after the injury. In the first series, after the operation, a continuous passive motion machine had been applied to the ankle, whereas in the second series, after surgery, the operated ankle joint had been immobilized in a splint or a cast. The aim of our study was to compare the clinical and radiographic results observed at follow-up in two series of patients who had been treated surgically using two different protocols. 


\section{Materials and methods}

From a pool of 45 patients with a unilateral bimalleolar or trimalleolar fracture, treated surgically between 1990 and 1995 at the Department of Orthopaedic Surgery of the University of Rome "Tor Vergata," we extracted 28 patients in whom we had applied a continuous passive motion machine to the operated ankle immediately after surgery. In all 28 patients, we had obtained an anatomical reduction that was very stable to passively exerted flexionextension movements. The remaining 17 cases had been considered unsuitable for CPM at the end of surgery because they had shown movements at the fracture site when the ankle was moved. All 28 patients were invited to our hospital for a clinical and radiographical evaluation: four could not be located, while two declined to participate in the study because they judged their follow-up useless, owing to the absence of any symptoms in the ankle. The remaining 22 patients made up the first series of patients. A comparative series of another 22 patients was selected from a different pool of 53 patients operated on between 1985 and 1990 in whom the operated ankle had been immobilized in a splint or in a cast after surgery. From this second pool of patients, we selected 32 fractures for which at surgery we had obtained an anatomical reduction and a stable internal fixation. We excluded the unstable fractures, evaluated at the end of surgery, from this second series. Ten patients either could not be located or declined to participate in the study, and the remaining 22 made up the second series of patients. The two series of patients were not randomized since the first series had been treated after 1990, whereas the second series had been treated before that date. In the first series of patients, 13 were female and 9 were male. Their age at injury ranged from 20 to 62 years, with an average of 41.8 years. The right ankle was involved in 15 cases and the left ankle in 7 . There were 8 bimalleolar fractures and 14 trimalleolar fractures. In 4 cases the fracture of the fibula was below the syndesmosis (Weber A), in 6 cases it was at the level of the syndesmosis (Weber B), whereas in 12 cases it was above the syndesmosis (Weber C). In one case an ankle dislocation was present, with a severe tibiofibular diastasis. In the second series, 10 were male and 12 were female; the average age at injury was 44.2 years, with a range between 18 and 69 years. The right ankle was involved in 13 cases and the left in 9. There were 10 bimalleolar and 12 trimalleolar fractures, classified in 2 cases as Weber A, in 8 cases as Weber B, and in 12 cases as Weber C.

The first series of patients had been treated surgically by anatomical open reduction and stable internal fixation (ORIF). The fibula was fixed by a plate and the medial malleolus by one cannulated screw. In 4 of the 12 trimalleolar fractures, we performed reduction and fixation of the posterior malleolus by two cannulated screws. In the remaining cases we did not synthesize the posterior bone fragment because it measured $<25 \%$ of the joint surface length, and it appeared to be well reduced at the end of the operation. The fracture of the fibula was always fixed first. In all 12 cases of Weber type $C$ fracture and in 1 case of type $\mathrm{B}$, the tibiofibular joint was diastased and we performed a trans-syndesmotic fixation with a screw going through one of the holes of the plate, in order to obtain an anatomical reduction of the diastasis and to further stabilize the osteosynthesis. Immediately after surgery a continuous passive motion machine was applied to the operated ankle, starting with $5^{\circ}$ of dorsiflexion and $10^{\circ}$ of plantar flexion. We gradually increased the passive movements of the ankle, in order to reach the maximum dorsiflexion and plantar flexion possible 2 weeks after the operation. The machine was applied full-time for 3 weeks, for the first three postoperative days at the hospital, and subsequently at home. Full-time means at least $8 \mathrm{~h}$ during the day and all night. The first day after surgery, patients were allowed to walk for short distances on crutches without weight bearing, wearing an elastic bandage. Gradual weight bearing was started 8 weeks after surgery. In all cases but one, the screw fixing the tibioperoneal syndesmosis was removed under local anesthesia before starting weight bearing.

The second series of patients had also been treated surgically by anatomical open reduction and internal fixation (ORIF), following the same criteria already described. The fibula was fixed first by a plate or intramedullary nail, while the medial malleolus was fixed with one screw. For the trimalleolar fractures, the posterior bone fragment was synthesized in six cases with one or two screws; in all these cases, it was $>25 \%$ of the entire articular surface. We performed a trans-syndesmotic fixation with one screw in all Weber $\mathrm{C}$ fractures. After surgery, all patients of this second series were immobilized in a splint or a cast for 3 weeks. The first day after surgery, patients were allowed to walk for short distances on crutches without weight bearing. Gradual weight bearing was started 8 weeks after surgery. In all cases, the screw fixing the tibioperoneal syndesmosis was removed under local anesthesia before starting weight bearing.

At follow-up, all patients were evaluated clinically, and radiographs of both ankles in AP and lateral views were taken. The AOFAS Ankle Hindfoot Score System [1] was used to rate the final clinical result. From the radiographic point of view, we evaluated the presence of signs of osteoarthritis of the ankle, with particular attention paid to the width of the superior space of the ankle mortise in the AP view.

The study was performed in accordance with the Declaration of Helsinki, it was approved by the Ethics Committee of our University Hospital, and informed 
Table 1 Results observed at follow-up in the two series of patients

\begin{tabular}{|c|c|c|c|c|c|c|}
\hline & $\begin{array}{l}\text { Number of } \\
\text { patients }\end{array}$ & $\begin{array}{l}\text { Length of } \\
\text { follow-up }\end{array}$ & $\begin{array}{l}\text { AOFAS score at } \\
\text { follow-up }\end{array}$ & $\begin{array}{l}\text { Sagittal motion of ankle } \\
\text { (flexion }+ \text { extension) } \\
\text { at follow-up }\end{array}$ & $\begin{array}{l}\text { Hindfoot motion } \\
\text { (inversion }+ \text { eversion) } \\
\text { at follow-up }\end{array}$ & $\begin{array}{l}\text { Osteoarthritis } \\
\text { (X-rays) at } \\
\text { follow-up }\end{array}$ \\
\hline \multirow[t]{2}{*}{$\begin{array}{l}\text { First } \\
\quad \text { series }\end{array}$} & \multirow[t]{2}{*}{22} & \multirow[t]{2}{*}{$10-14$ years } & $\begin{array}{l}95.7 \text { points } \\
\quad(87-100 \text { points })\end{array}$ & $52^{\circ}\left(30-70^{\circ}\right)$ & $50^{\circ}\left(44-55^{\circ}\right)$ & \multirow[t]{2}{*}{2 cases } \\
\hline & & & SD 3.42 & SD 9.86 & SD 3.30 & \\
\hline \multirow[t]{2}{*}{$\begin{array}{l}\text { Second } \\
\text { series }\end{array}$} & \multirow[t]{2}{*}{22} & \multirow[t]{2}{*}{ 14-20 years } & $\begin{array}{l}88 \text { points } \\
\quad \text { (68-100 points) }\end{array}$ & $34^{\circ}\left(20-65^{\circ}\right)$ & $44^{\circ}\left(32-53^{\circ}\right)$ & \multirow[t]{2}{*}{7 cases } \\
\hline & & & SD 10.60 & SD 11.01 & SD 6.59 & \\
\hline
\end{tabular}

consent was obtained from all the patients included in the study.

A statistical analysis was performed using the MannWhitney method. At $P<0.05$, the difference or correlation was considered significant.

\section{Results}

First series

Follow-up ranged from 10 to 14 years, with an average of 11.5 years. At follow-up, the score ranged from 87 to 100 points (average 95.7 points, standard deviation 3.42 points); in these patients, the sagittal motion of the ankle (flexion plus extension) measured from $30^{\circ}$ to $70^{\circ}$, with an average of $52^{\circ}$ (standard deviation $9.86^{\circ}$ ). The hindfoot motion (inversion plus eversion) measured between $44^{\circ}$ and $55^{\circ}$, with an average of $50^{\circ}$ (standard deviation $3.30^{\circ}$ ) (Table 1) (Fig. 1). At radiographic examination, minor or moderate signs of osteoarthritis were present in two cases. In one patient, who had had a fracture-dislocation with severe diastasis of the tibiofibular syndesmosis, we observed moderate signs of osteoarthritis, including a narrowing of the superior joint space of the ankle mortise that measured $1.4 \mathrm{~mm}$. Moreover, ectopic bone had formed below the tip of the medial malleolus, and on the anterior border of the distal tibial epiphysis. However, at clinical examination the patient was pain-free, and the function and alignment of the ankle were normal. The other patient was an amateur soccer player. He complained of mild pain in the ankle only at the end of a soccer match, which resolved spontaneously in about $1 \mathrm{~h}$. In this patient, X-rays taken at follow-up showed minor signs of osteoarthritis, with a narrowing of the superior joint space of the ankle mortise that measured $1.5 \mathrm{~mm}$. In one patient, the screw going through the syndesmosis broke 2 months after starting weight bearing and we removed it. In the remaining cases, we always removed the trans-syndesmotic screw before starting weight bearing.
The complications observed in this series were in one case a breakage of the trans-syndesmotic screw, which occurred 2 months after full weight bearing, and a superficial wound infection, which was successfully treated with antibiotic therapy.

Second series

The follow-up ranged from 14 to 20 years, with an average of 15.8 years. At follow-up, the AOFAS score ranged from 68 to 100 points (average 88 points, standard deviation 10.60 points). The sagittal motion of the ankle (flexion plus extension) measured from $20^{\circ}$ to $65^{\circ}$, with an average of $34^{\circ}$ (standard deviation $11.01^{\circ}$ ), while the hindfoot motion (inversion plus eversion) measured between $32^{\circ}$ and $53^{\circ}$, with an average of $44^{\circ}$ (standard deviation $6.59^{\circ}$ ) (Table 1). At radiographic examination, in six patients we noticed minor signs of osteoarthritis of the ankle joint, with narrowing of the superior joint space of the ankle mortise that measured from 1.2 to $2 \mathrm{~mm}$ (Fig. 2). In another patient, X-rays showed severe osteoarthritis of the ankle joint. The complications which occurred in this series were in one case a superficial wound infection and in another case a deep wound infection, both successfully treated with antibiotic therapy; in another case we observed a deep vein thrombosis (DVT), which was successfully treated with low-molecular-weight heparin for 2 months.

The difference between the two series of patients as regards the final score observed at follow-up was statistically significant ( $P$ value 0.01 ), while the difference for the values of the range of motion of the ankle was not statistically significant ( $P$ value 0.12 ); the difference for the values of the range of motion of the hindfoot was also not statistically significant $(P$ value 0.35$)$.

\section{Discussion}

The prognosis of bimalleolar and trimalleolar ankle fractures is strictly related to the quality of the reduction; for 
Fig. 1 Anteroposterior radiograph of a Weber $\mathrm{C}$ trimalleolar fracture of the ankle in a 48-year-old patient (a), treated surgically by open reduction and internal fixation (ORIF). Immediately after surgery a CPM machine was applied to the operated ankle. Six months later, the fractures had healed perfectly (b). At follow-up, 10 years later, the patient had a complete range of motion of the ankle, with minor radiographic signs of osteoarthritis (c-d)
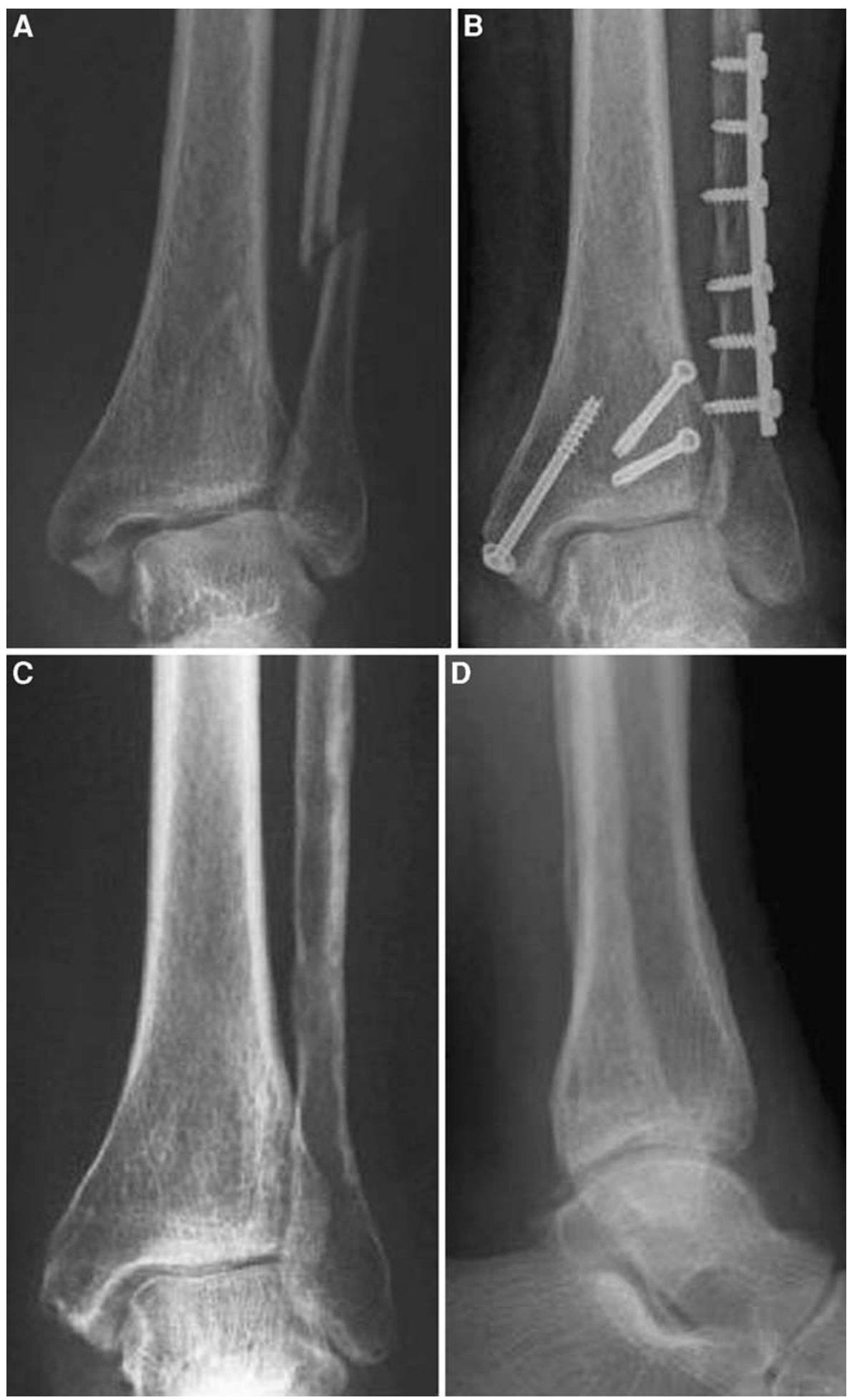

this reason, in order to obtain a satisfactory final result in all displaced fractures, even in mild displacement, it is necessary to perform an anatomical open reduction and a stable internal fixation. Inappropriate reduction of these fractures will cause ankle instability and early degenerative osteoarthritis of the joint [9]. The reduction of the lateral malleolus significantly influences the outcome of the operation. In fact, as reported by other authors, the anatomical reduction of the lateral malleolus plays a key role in the surgical treatment of these lesions [10-12]. In accordance with this principle, in all cases reported in this study we performed first the open reduction of the lateral 
Fig. 2 Anteroposterior radiograph of a Weber $\mathrm{C}$ trimalleolar fracture of the ankle in a 34-year-old patient (a), treated surgically by ORIF (b). After surgery, a plaster cast was applied to the operated ankle. At follow-up, 18 years later, the patient had a complete range of motion of the ankle, although the radiographs showed minor signs of osteoarthritis of the ankle, with narrowing of the superior space of the ankle mortise that measured $1.4 \mathrm{~mm}$ (c-d)
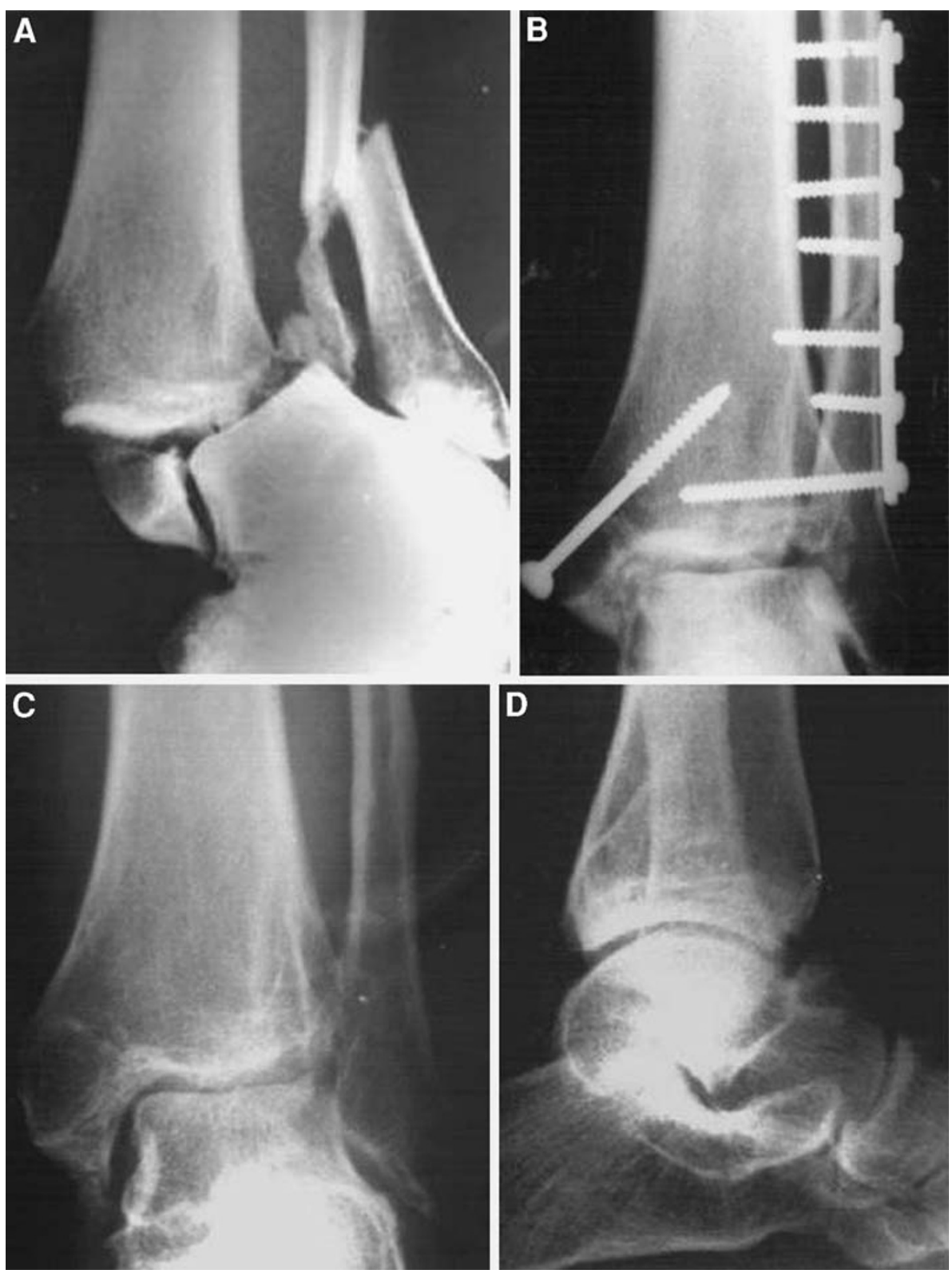

malleolus. Several authors recommend use of a syndesmotic screw in Weber type $C$ fractures [10, 13-17]. Other authors have suggested avoiding trans-syndesmotic fixation of Weber type $\mathrm{C}$ fractures when rigid fixation of the medial and lateral fractures is achieved or when the fibular fracture is within $4.5 \mathrm{~cm}$ of the joint and there is a deltoid ligament tear $[18,19]$. In one Weber type B and in all Weber type C fractures of our two series, we applied a trans-syndesmotic screw to obtain an adequate reduction of the tibiofibular diastasis and to further stabilize the osteosynthesis. In fact, we believe that the use of a trans-syndesmotic screw is always indicated in Weber type $\mathrm{C}$ fractures, because this supplemental fixation gives an immediate, better stabilization of the synthesis with better healing of the tibiofibular ligaments, any looseness of which might entail later instability of the ankle, with the development of early degenerative joint disease. Moreover, in the treatment protocol of our first series, a good stabilization of the synthesis is mandatory to permit the immediate application of the passive motion machine. Some authors have reported limitation of ankle dorsiflexion following application of a syndesmotic screw, suggesting therefore removal of the screw 6-8 weeks after surgery in an attempt to restore full ankle motion [20, 21]. In agreement with these authors, we removed the trans-syndesmotic screw 8 weeks after surgery in all cases but one, in which the screw broke 2 months after full weight bearing.

At follow-up, we never observed significant restriction of the ankle motion in our first series, whereas a moderate restriction of the sagittal motion and/or the hindfoot motion 
was observed in seven patients of our second series. In fact, the average of the range of motion of the ankle measured $52^{\circ}$ in the first series and $34^{\circ}$ in the second, while the average of the range of motion of the hindfoot was $50^{\circ}$ in the first series and $44^{\circ}$ in the second series, although the difference between the two series of patients was not statistically significant.

Some authors have reported that osteosynthesis of the posterior malleolus is indicated only when the bone fragment is $>25 \%$ of the joint surface [6, 22]. In accordance with these authors, we performed synthesis of the posterior malleolus in four cases of the first series and in six cases of the second series, in which the fragment was $>25 \%$ of the articular surface. At follow-up, we observed similar results in all of the trimalleolar fractures.

In our first series of patients, immediately after surgery we applied a continuous passive motion machine to all of the operated ankles. To the best of our knowledge, no reports have been previously published on the immediate postoperative application of a passive motion machine for the postoperative treatment of these lesions, although Ahl et al. [5] have recommended active early mobilization of operated-on ankle fractures, starting the second week after the operation. Moreover, Salter et al. [23] reported in an experimental study that early joint motion stimulates articular cartilage to heal by means of a reparative tissue more similar to hyaline cartilage than to fibrocartilage. We believe that postoperative passive mobilization may enable the patient to achieve quick recovery of the ankle range of motion, as well as better healing of the articular cartilage lesions. In fact, none of the patients in our first series had significant restriction of range of motion of the ankle joint at follow-up, compared with the second series in which we observed moderate limitation of the ankle joint in seven patients. At radiographic examination, we observed minor or moderate signs of osteoarthritis in only two patients of the first series, one of whom had suffered extensive damage to the articular cartilage owing to a severe fracture-dislocation with disruption of the tibiofibular syndesmosis, whereas we observed minor or severe signs of osteoarthritis of the ankle joint in seven patients of the second series.

Some authors [24, 25] have reported a high risk of postoperative wound complications in patients treated for ankle fractures with surgery followed by early mobilization. Inasmuch as we observed a similar number of infections in each of our two series of patients, we did not find any correlation between wound complications and early mobilization of the ankle.

In conclusion, we believe that, in ankle fractures, continuous passive motion starting immediately after surgery allows good recovery of range of motion of the ankle and could minimize risk of osteoarthritis.
Conflict of interest statement The authors declare that they have no commercial associations that might pose a conflict of interest in connection with the submitted manuscript.

\section{References}

1. Kitaoka HB, Alexander IJ, Adelaar RS et al (1994) Clinical rating system for the ankle-hindfoot, midfoot, hallux, and lesser toes. Foot Ankle 15:349-353

2. Hughes JL, Weber H, Willenegger H, Kuner EH (1979) Evaluation of ankle fractures: nonoperative and operative treatment. Clin Orthop 138:111-119

3. Joy G, Patzakis MJ, Harvey JP (1974) Precise evaluation of the reduction of severe ankle fractures. J Bone Joint Surg 65A:979993

4. Michelson JD (1995) Current concepts review. Fractures about the ankle. J Bone Joint Surg 77A:142-152

5. Ahl T, Dalen N, Lundberg A, Bylund C (1993) Early mobilization of operated on ankle fractures. Prospective, controlled study of 40 bimalleolar cases. Acta Orthop Scand 64(1):95-99

6. Beris AE, Kabbani KT, Xenakis TA et al (1997) Surgical treatment of malleolar fractures. A review of 144 patients. Clin Orthop 341:90-98

7. Dogra AS, Rangan A (1999) Early mobilization versus immobilization of surgically treated ankle fractures. Prospective randomized control trial. Injury 30:417-419

8. Egol KA, Dolan R, Koval KJ (2000) Functional outcome of surgery for fractures of the ankle. J Bone Joint Surg 82B:246249

9. Rockwood CA, Green DP, Bucholz RV (1991) Fractures in adults. JB Lippincott Co, Philadelphia, pp 1983-2039

10. Leeds HC, Ehrlich MG (1984) Instability of the distal tibiofibular syndesmosis after bimalleolar ankle fractures. J Bone Joint Surg 66A:490-503

11. Wanders L, Oliver CW (1998) Fibular malreduction in $\mathrm{AO} /$ Weber type C ankle fractures. Injury 29:144-146

12. Yablon IG, Heller FG, Shouse L (1977) The key role of the lateral malleolus in displaced fractures of the ankle. J Bone Joint Surg 59A:169-173

13. Chissel HR, Jones J (1995) The influence of a diastasis screw on the outcome of Weber type-C ankle fractures. J Bone Joint Surg 77B:435-438

14. De Souza IJ, Gustilo RB, Meyer TJ (1985) Results of operative treatment of displaced external rotation abduction fractures of the ankle. J Bone Joint Surg 67A:1066-1073

15. Kaye RA (1989) Stabilization of ankle syndesmosis injuries with a syndesmosis screw. Foot Ankle 9:290-293

16. Purvis GD (1982) Displaced, unstable ankle fractures. Classification, incidence and management of a consecutive series. Clin Orthop 165:91-98

17. Riegels-Nielsen P, Christensen J, Greiff J (1983) Destability of the tibiofibular syndesmosis following rigid internal fixation for type $\mathrm{C}$ malleolar fractures: an experimental and clinical study. Injury 14:357-360

18. Boden SD, Labropoulos PA, McCowin P, Lestini WF, Hurwithz SR (1989) Mechanical consideration for the syndesmosis screw. A cadaver study. J Bone Joint Surg 71A:1548-1555

19. Yamaguchi K, Martin CH, Boden SD, Labropoulos PA (1994) Operative treatment of syndesmotic disruptions without use of a syndesmotic screw: a prospective clinical study. Foot Ankle 15:407-413

20. Needleman RL, Skrade DA, Stiehl JB (1989) Effect of the syndesmotic screw on ankle motion. Foot Ankle 10:17-24 
21. Olerud C (1985) The effect of the syndesmotic screw on the extension capacity of the ankle joint. Arch Orthop Trauma Surg 104:299-302

22. Broos PLO, Bisschop APG (1991) Operative treatment of ankle fractures in adults: correlation between types of fracture and final results. Injury 22:403-406

23. Salter RB, Simmonds DF, Malcolm BW (1980) The biological effect of continuous passive motion on the healing of full thickness defects in articular cartilage. J Bone Joint Surg 62A:1232-1251
24. Vioreanu M, Dudeney S, Hurson B, Kelly E, O'Rourke K, Quinlan W (2007) Early mobilization in a removable cast compared with immobilization in a cast after operative treatment of ankle fractures: a prospective randomized study. Foot Ankle Int 28(1):13-19

25. Lehtonen H, Jarvinen T, Honkonen S, Nyman M, Vihtonen K, Jarvinen M (2003) Use of a cast compared with a functional ankle brace after operative treatment of an ankle fracture. J Bone Joint Surg 85A:205-211 